\title{
Bronchoscope Tracking Without Fiducial Markers Using Ultra-tiny Electromagnetic Tracking System and Its Evaluation in Different Environments
}

\author{
Kensaku Mori ${ }^{1,2}$, Daisuke Deguchi ${ }^{2}$, Kazuyoshi Ishitani ${ }^{1}$, \\ Takayuki Kitasaka ${ }^{1,2}$, Yasuhito Suenaga ${ }^{1,2}$, Yosihnori Hasegawa ${ }^{3,2}$, \\ Kazuyoshi Imaizumi ${ }^{3}$, and Hirotsugu Takabatake ${ }^{4}$ \\ ${ }^{1}$ Graduate School of Information Science, Nagoya University, \\ Furo-cho, Chikusa-ku, Nagoya, Aichi, 464-8603, Japan \\ kensaku@is.nagoya-u.ac.jp \\ ${ }^{2}$ Innovative Research Center for Preventive Medical Engineering, Nagoya University \\ ${ }^{3}$ Graduate School of Medicine, Nagoya University \\ ${ }^{4}$ Sapporo Minami-Sanjyo Hospital
}

\begin{abstract}
This paper presents a method for bronchoscope tracking without any fiducial markers using an ultra-tiny electromagnetic tracker (UEMT) for a bronchoscopy guidance system. The proposed method calculates the transformation matrix, which shows the relationship between the coordinates systems of the pre-operative CT images and the UEMT, by registering bronchial branches segmented from CT images and points measured by the UEMT attached at the tip of a bronchoscope. We dynamically compute the transformation matrix for every pre-defined number of measurements. We applied the proposed method to a bronchial phantom in several experimental environments. The experimental results showed the proposed method can track a bronchoscope camera with about $3.3 \mathrm{~mm}$ of target registration error (TRE) for wood table environment and $4.0 \mathrm{~mm}$ of TRE for examination table environment.
\end{abstract}

\section{Introduction}

A bronchoscope is a flexible endoscope that is a tool to observe the inside of the bronchus. A physician inserts a bronchoscope into a patient airway and diagnoses the inside of it with watching a TV monitor showing video images captured by the camera. Since the bronchus has complex tree structure, a physician easily gets disoriented during bronchoscopy. It is strongly expected to develop a bronchoscopy guidance system that assists a physician during bronchoscopy.

In construction of a bronchoscopy guidance system, a function for bronchoscope tracking is quite important. There are two types of methods in bronchoscope tracking: (a) methods based on image registration and (b) methods using trackers (positional sensors). Bronchoscope tracking based on image registration basically tracks the tip of the real bronchoscope (RB) by finding a 
virtual bronchoscopic (VB) image that is the most similar to the current frame of the real bronchoscope. VB images are generated from CT images. Mori et al. reported a method for tracking a bronchoscope using epipolar geometry analysis and intensity-based image registration of real and virtual endoscopic images [1. However, image-based registration is very weak against occlusion of RB views.

Methods for bronchoscope tracking using positional trackers try to directly capture the motion of the RB camera by attaching trackers at the tip of the bronchoscope. They are very robust to occlusion of views. Nowadays, ultra-tiny electromagnetic trackers (UEMT) are now available from several companies. These trackers can be inserted into the working channel of the bronchoscope. Schneider et al. used a UEMT sensor to obtain the bronchoscope camera position 2. Their registration errors were about $4 \mathrm{~mm}$ at the trachea and $15 \mathrm{~mm}$ at the right upper lobe bronchus. Wegner et al. also tried to track a bronchoscopy by UEMT, but details and performance are not presented in 3 . Deligianni et al. proposed bronchoscope tracking using the UEMT sensor, and $p q$-based registration techniques are applied to improve tracking accuracy [4.

In general, most of surgical navigation systems utilizing a tracker require fiducial markers attached on a patient's body for registering the coordinate system (CS) of a pre-operative image (i.e. CT images) and the CS of the tracker. In the case of bronchoscopy, effective placement of fiducial markers is impossible, since we need to place fiducial markers inside the bronchus for better tracking performance.

This paper tries to develop a method for tracking an RB camera by using a UEMT tracker. Here, we develop a fiducial-marker free algorithm that registers the CSs of a tracker and pre-operative CT images. The proposed method calculates the transformation matrix showing the relationship between the CSs of the pre-operative CT image and the UEMT by dynamically finding the relation between bronchial branches segmented from CT images and the positions of the RB tip. Also, we evaluate the proposed method in different environments including a wood table and a real examination table.

\section{Method}

\section{$2.1 \quad$ Overview}

We attach the sensing coil of a UEMT at the tip of the bronchoscope. The location of the sensing coil is very close to the RB camera. We assume that the RB camera moves only inside the bronchus and moves along the medial axis of the bronchus. We calculate the transformation matrix showing the relationship between the CSs of the UEMT and the pre-operative CT images by registering bronchial branches acquired from the CT images and the points measured by the UEMT. In this method, the transformation matrix showing the relationship between two CSs are gradually updated as the bronchoscope moves inside the bronchus. Points used in registration are also dynamically updated. 




Fig. 1. Illustration showing the relationship between coordinate systems

\subsection{Definition of Coordinate Systems}

Figure 1 shows the relationship between each CS. We denote a position in the CS of the RB camera, $C$, as $\boldsymbol{p}_{C}$. The position of the corresponding point in the CT CS is described as $\boldsymbol{p}_{C T}$. We consider the CS defined by the sensor attached on a patient body for tracking patient movement (usually called $D R F$ ) as the world CS $W$. The position of the point $\boldsymbol{p}_{C}$ in the world CS is described as $\boldsymbol{p}_{W}$. At time $k$, the relationship between $\boldsymbol{p}_{C}$ and $\boldsymbol{p}_{C T}$ is described as

$$
\begin{aligned}
\boldsymbol{p}_{C T} & ={ }_{W}^{C T} \mathbf{T}{ }_{F}^{W} \mathbf{T}_{S}^{F} \mathbf{T}^{k}{ }_{C}^{S} \mathbf{T} \boldsymbol{p}_{C}={ }_{W}^{C T} \mathbf{T}{ }_{S}^{W} \mathbf{T}^{k}{ }_{C}^{S} \mathbf{T} \boldsymbol{p}_{C}={ }_{W}^{C T} \mathbf{T}\left(\begin{array}{cc}
{ }_{S} \mathbf{R}^{k}{ }^{W} \boldsymbol{t}_{S}^{k} \\
\mathbf{0}^{T} & 1
\end{array}\right){ }_{C}^{S} \mathbf{T} \boldsymbol{p}_{C} \\
& ={ }^{C T} \mathbf{T} \boldsymbol{p}_{W}^{k},
\end{aligned}
$$

where the sensor CS $S$ is a CS defined by the UEMT sensor attached at the RB camera and ${ }_{C}^{S} \mathbf{T}$ is the transformation matrix from CS $C$ to $S$. The CS of the magnetic field generator (MFG) of the UEMT is described as $F$. Transformation matrix ${ }_{F}^{W} \mathbf{T}$ is the transformation from UEMT CS $F$ to the world CS $W$, and ${ }_{W}^{C T} \mathbf{T}$ is the transformation matrix from the world CS $W$ to the CT CS. Transformation from $S$ to $W$ is given by ${ }_{S}^{W} \mathbf{T}^{k}={ }_{F}^{W} \mathbf{T}_{S}^{F} \mathbf{T}^{k}$. In Eq. (11), ${ }_{S}^{W} \mathbf{R}^{k}$ and ${ }^{W} \boldsymbol{t}_{S}^{k}$ are the rotation matrix and transformation vector measure by the UEMT sensor at time $k$, respectively. Transformation ${ }_{C}^{S} \mathbf{T}$ shows the relationship between the CSs $C$ and $S .{ }_{C}^{S} \mathbf{T}$ can be obtained when the sensor is attached at the tip of the RB. The position and the orientation of the RB camera in the world CS $W$ can be obtained from the output of the UEMT and ${ }_{C}^{S} \mathbf{T}$. By finding the transformation ${ }_{W}^{C T} \mathbf{T}$, it is possible to register the CSs $C$ and $C T$. In this paper, we use the coordinate system $C T$ as the CS of the VB camera.

\subsection{Bronchial Tree Representation}

The proposed method uses bronchial branches extracted from pre-operative CT images to register CSs. Here, we represent the $i$-th bronchial branch as $\mathbf{b}_{i}=$ $\left\{\boldsymbol{b}_{i}^{s}, \boldsymbol{b}_{i}^{e}\right\}$, where $\boldsymbol{b}_{i}^{s}$ and $\boldsymbol{b}_{i}^{e}$ are the start and the end positions of $\mathbf{b}_{i}$ on the $C T$ CS. Also, we represent a set of bronchial branches $\mathcal{B}=\left\{\mathbf{b}_{i} \mid i=1, \ldots, n\right\}$, where $n$ is the number of bronchial branches. 


\subsection{Processing Procedure}

Extraction of bronchial branches. To obtain bronchial branches from CT images, we utilize Kitasaka's method [5]. This method can extract bronchi regions and their tree representations, simultaneously.

Estimation of ${ }_{W}^{C T} \mathbf{T}$. During bronchoscopy, we obtain ${ }_{S}^{W} \mathbf{T}^{k}$ from the $k$-th output of the UEMT attached at the tip of the bronchoscope. The RB camera position $\boldsymbol{p}_{W}^{k}$ at time $k$ in the world coordinate system is computed by

$$
\boldsymbol{p}_{W}^{k}={ }_{S}^{W} \mathbf{T}_{C}^{k S} \mathbf{T} \boldsymbol{p}_{C} .
$$

During bronchoscopy, we record a set of the RB camera positions $\mathcal{P}=\left\{\boldsymbol{p}_{W}^{k}\right\}$ $\left(k=1, \ldots, N_{1}\right)$. The $l$-th updated transformation matrix is denoted as ${ }_{W}^{C T} \mathbf{T}^{l}$. Updates are performed for every pre-defined number of measurements. The set of the RB camera position used for $l$-th update of ${ }_{W}^{C T} \mathbf{T}^{l}$ is represented as $\mathcal{P}^{l} . \tilde{\mathcal{P}}$ and $\hat{\mathcal{P}}$ are temporary sets to store the RB camera positions. $\mathcal{Q}$ is the set of positions corresponding to the $\boldsymbol{p}_{W}^{k}$ on bronchial branches $\mathcal{B}$. The procedure for computing ${ }_{W}^{C T} \mathbf{T}$ is described in below.

\section{Procedure for estimating matrix ${ }_{W}^{C T} \mathbf{T}$}

[Step 1] Initialize variables as $k=1, l=0$, and $\mathcal{P}^{0}=\phi$.

[Step 2] $\tilde{\mathcal{P}}=\phi$ and $l=l+1$.

[Step 3] Compute $\boldsymbol{p}_{W}^{k}$ using output ${ }_{S}^{W} \mathbf{T}^{k}$ of the UEMT sensor by Eq. (2) and append them to temporary set $\tilde{\mathcal{P}}$.

[Step 4] $k=k+1$.

[Step 5] Repeat Steps 3 and 4 until $\left|\mathcal{P}^{l-1} \cup \tilde{\mathcal{P}}\right| \geq N_{1}$ is satisfied. ( $\cup$ : the set union. $|\mathcal{A}|$ : the number of elements included in the set $\mathrm{A}$ )

[Step 6] Compute $\mathcal{P}^{l}$ as $\mathcal{P}^{l}=\mathcal{P}^{l-1} \cup \tilde{\mathcal{P}}$.

[Step 7] Initialize ${ }_{W}^{C T} \mathbf{T}^{l}$ as ${ }_{W}^{C T} \mathbf{T}^{l}={ }_{W}^{C T} \mathbf{T}^{l-1}$

[Step 8] We compute ${ }_{W}^{C T} \mathbf{T}^{l}$ by the well-known ICP-like algorithm (called ICB algorithm). The difference from original is that we are measuring the distance between a point and a tree structure (branches). The inputs of the ICB algorithms are (a) a set of points $\mathcal{P}^{l}$ and (b) a set of branches $\mathcal{B}$. The distance between a point and a branch is calculated by finding the closest branch to the target point and computing the minimum distance between the selected branch and the target point. The ICB

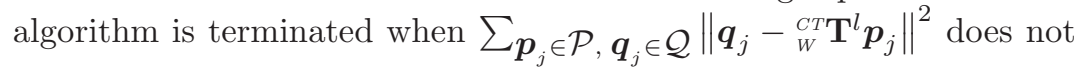
change. Here, $\mathcal{Q}$ is a set of points $\boldsymbol{q}_{j}$ that are corresponding to $\boldsymbol{p}_{j}$ and are on the selected branch,

[Step 9] Reduce the number of elements included in the set $\mathcal{P}^{l}$ into $N_{2}$ by taking the following procedures:(a) Select a point whose transformed position is closest for each branch. (b) Store the selected point $\hat{\mathcal{P}}$. (c) Repeat (a) and (b) while $|\hat{\mathcal{P}}|<N_{2}$.

[Step 10] $\mathcal{P}^{l}=\hat{\mathcal{P}}$. Return to Step 3 . 


\section{$3 \quad$ Experiments and Results}

We applied the proposed method to a rubber bronchial phantom. Two different UEMTs, the microBird (Tracker1) and the 3D-Guidance (Tracker2) (Assention Technology Inc., Burlington, VT, USA) are used. Tracker1 has a cubic MFG and Tracker2 has a flatbed MFG that can be placed underneath a patient, respectively. Sensing coils are inserted into the working channel of the bronchoscope BF-200 (Olympus, Tokyo). For each tracker, we evaluated the proposed method in two types of environments: (a) a wood table and (b) a real examination table (see Table 1) Also, we utilized a method for compensating UEMT outputs (called tracker compensation) [6].

To evaluate the stability and effectiveness of the proposed method, we used a rubber bronchial phantom. CT images of the phantom were taken by a multidetector CT scanner $(512 \times 512$ pixels, 341 slices, $0.684 \mathrm{~mm}$ resolution on a slice image, $1.25 \mathrm{~mm}$ of X-ray collimation, $0.5 \mathrm{~mm}$ of reconstruction pitch.) Bronchial region is segmented by Kitasaka's method [5]. In experiments, we inserted the $\mathrm{RB}$ into several branches including the trachea, the right main bronchus, and the left main bronchus. We obtained 1000 pairs of UEMT outputs and RB images.

The transformation matrix ${ }_{W}^{C T} \mathbf{T}^{l}$ was dynamically updated under the parameters of $N_{1}=200$ and $N_{2}=180$. In real bronchoscopy, the posture of the RB at the trachea is almost same for all patients. The algorithm initialized ${ }_{W}^{C T} \mathbf{T}^{0}$ by using the output of the UEMT at the trachea. The system shows a virtual bronchoscopic view at the carina (first bifurcation). An operator inserts a bronchoscope to this point and set up a bronchoscope to take a real bronchoscopic view similar to virtual bronchoscopic view. By pushing a button, the system calculates ${ }_{W}^{C T} \mathbf{T}^{0}$. Figure 5 shows the results of the proposed method in examination table environment with Tracker2. UEMT compensation technique was used in this case. Attached video was captured in a wood table environment with Tracker2 of no compensation. Intrinsic parameters of the RB camera obtained by Zhang's method are used to generate VB images. We measured target registration error (TRE) to evaluate accuracy of the proposed method. During bronchoscopy. Two kind of TRE, (a) Internal TRE and (b) external TRE, were measured. For internal TRE, we held the RB camera at five branching points. Then, the RB camera position $\boldsymbol{p}_{W}^{i}(i=1, \ldots, 5)$ was computed from the outputs of the UEMT. On the other hands, the positions of the corresponding points $\boldsymbol{p}_{C T}^{i}(i=1, \ldots, 5)$ on $\mathrm{CT}$ images are manually identified by three engineers. The averaged positions are used as gold standard. Internal TRE at the $l$-th update $\left(E r r^{l}\right)$ was calculated by $E r r^{l}=\frac{1}{5} \sum_{i=1}^{5}\left\|\boldsymbol{p}_{C T}^{i}-{ }_{W}^{C T} \mathbf{T}^{l} \boldsymbol{p}_{W}^{i}\right\|$. In calculation of external TRE, we used 18 fiducials allocated outside of the bronchus phantom. The points of these fiducials were also measured by the UEMT and were measured on CT images. Then, external TREs were calculated in same way. Internal TRE measurements were performed in four different environments shown in Table 1 Results are also presented in the same table. Figure 3 shows TREs measured in different experimental environments. Figure 4 shows external TREs measured in Environment 3 without compensation with changing $N_{2}$ for fixed $N_{1}=200$. 


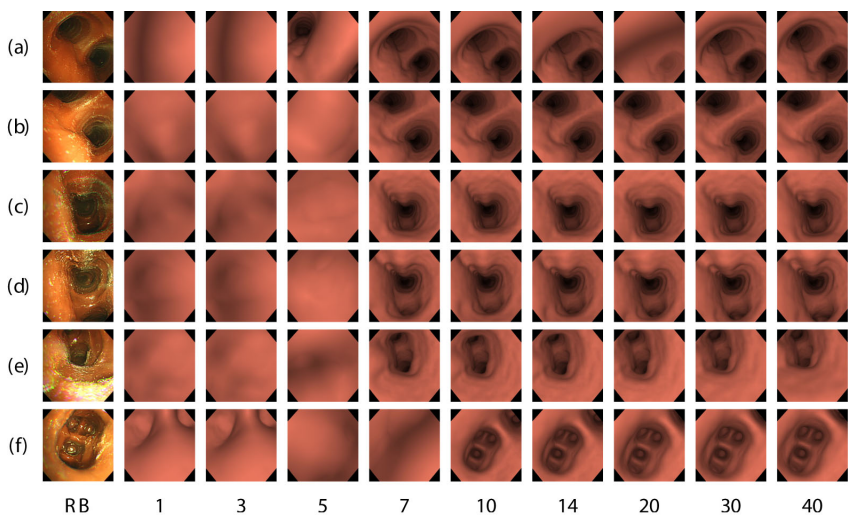

Fig. 2. Results of camera tracking. The top row shows the RB images at four positions of the phantom: the trachea, the left main bronchus, the right lower lobe bronchus, and the left lower lobe bronchus. The bottom row shows VB images generated using transformation matrix ${ }_{W}^{C T} \mathbf{T}^{40}$, which is estimated by the proposed method (Tracker2 / Examination table / Output Compensation).

Table 1. TREs for each experimental environments (Env.: Environment)

\begin{tabular}{|c|c|c|c|c|c|}
\hline \multirow[t]{2}{*}{ Env. } & \multirow[t]{2}{*}{ Equipments } & \multicolumn{2}{|c|}{$\begin{array}{l}\text { Min. Internal } \\
\text { TRE (mm) }\end{array}$} & \multicolumn{2}{|c|}{$\begin{array}{c}\text { Max. Internal } \\
\text { TRE }(l>10)(\mathrm{mm})\end{array}$} \\
\hline & & w/o Comp. & $\mathrm{w} / \operatorname{Comp}$ & $\mathrm{w} / \mathrm{o}$ Comp. & $\mathrm{w} / \mathrm{Com}$. \\
\hline Env.1 & Tracker1/Wood table & 4.5 & $\overline{4.6}$ & $\overline{5.7}$ & $\overline{7.2}$ \\
\hline Env.2 & Tracker1/Examination table & 4.4 & 6.1 & 6.9 & 8.3 \\
\hline Env.3 & Tracker2/Wood table & 3.3 & 3.4 & 4.7 & 4.2 \\
\hline Env.4 & Tracker2/Examination table & 3.9 & 4.0 & 5.2 & 5.2 \\
\hline
\end{tabular}

\section{Discussion}

As shown in Fig. 2, VB images generated from the transformation matrix ${ }_{W}^{C T} \mathbf{T}^{40}$ are quite similar to RB images. This figure shows the proposed method can properly register the coordinate systems of CT image and the UEMT. The initial TREs of the transformation matrix ${ }_{W}^{C T} \mathbf{T}^{0}$ are about $15 \mathrm{~mm}$ to $30.0 \mathrm{~mm}$. However, they gradually decrease as enough number of updates are performed (Fig. 3). For example, the TREs take minimum values of $3.3 \mathrm{~mm}$ at $l=38$ in Environment 3 and $3.8 \mathrm{~mm}$ at $l=11$ in Environment 4, respectively. This result is almost same or better than Schneider's experiment 2]. Although Schneider's method requires fiducial markers or reference positions to obtain the RB camera position, the proposed method requires no fiducial marker. This is great advantage of the proposed method, because it is quite difficult to place fiducial markers inside the bronchus. From Fig. 4 we can say that errors are relatively small for larger $N_{2}$. This is because we use global information for larger $N_{2}$. Since we tested the proposed method by only using a static phantom. it is required to 


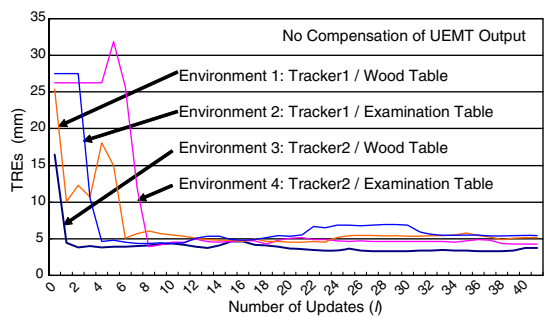

Fig. 3. Internal TREs of estimated ${ }_{W}^{C T} \mathbf{T}^{l}$

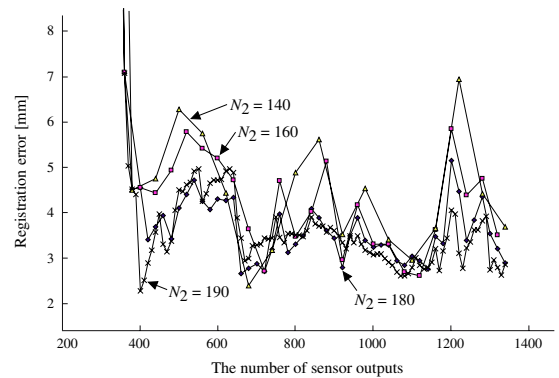

Fig. 4. External TREs of estimated ${ }_{W}^{C T} \mathbf{T}^{l}$ with fixing $N_{1}=200$ and changing $N_{2}$ (a)



(b)

(c)

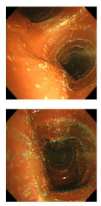

(d)

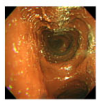

(e)

(f)



$\mathrm{RB}$


1
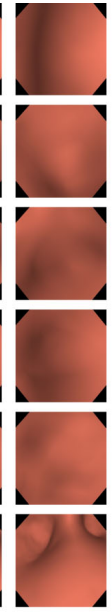

3
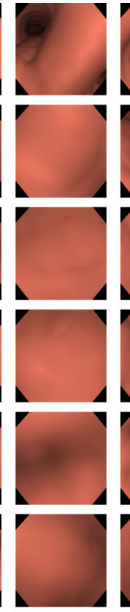

5
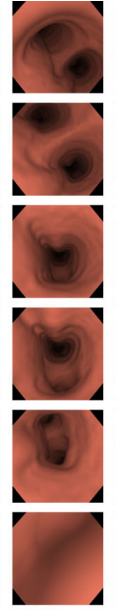

7
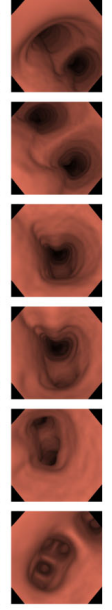

10
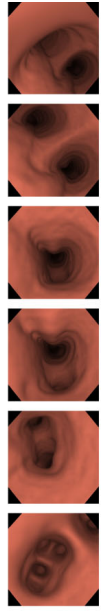

14
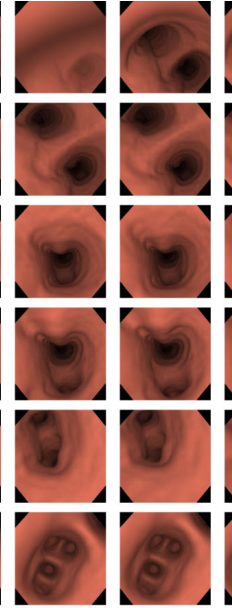

30
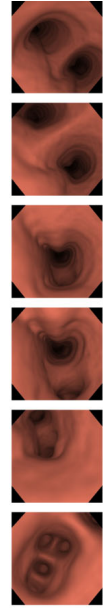

40

Fig. 5. Results of VB images generated by transformation matrix ${ }_{W}^{C T} \mathbf{T}^{l}$ at different branching points when number of update $l$ increases

evaluate by using dynamic phantom simulating breathing motion. Also, we need to investigate influence of deformation of the bronchus in future experiments.

We obtained UEMT sensor output by inserting the RB camera in the order of trachea, the right main bronchus, the left main bronchus, the right upper lobe bronchus, the right lower lobe bronchus, and the left lower lobe bronchus. In Fig. 5, the transformation matrix ${ }_{W}^{C T} \mathbf{T}$ is calculated from only UEMT outputs around the trachea and the right main bronchus for low $l$. Since the RB camera goes through the right main bronchus in later updates, VB images generated by the $\mathrm{RB}$ camera positions in the $C T$ CS become very close to the RB images in later updates.

As seen in Table1, the proposed method worked well in several environments. Even in the environment using the examination table, the proposed method can track the RB camera in error of $5 \mathrm{~mm}$ or less. Compensation of UEMT outputs 
did not improve tracking performance. Since the proposed method registers measured points to the medial axis of the bronchus, the proposed method can correct sensing errors of the UEMT by geometric constraints (bronchial branches.) The compensation method affected worse. Tracking performance of Tracker 2 was better than that of Tracker1.

We assumed that bronchial branches are accurately segmented by Kitasaka's method. Therefore, the proposed method cannot estimate the correct ${ }_{W}^{C T} \mathbf{T}^{l}$ if extraction of bronchial branches fails. Also, the proposed method estimates transformation matrix by assuming that the RB camera moves on the medial line of the bronchi region or almost at the center of the bronchi region. Precise evaluation about this assumption should be performed. Also, the proposed method requires that a bronchoscope should be inserted into the right and left lungs. We think this requirement is also acceptable, since a bronchoscopist normally inserts a bronchoscope into both sides of the bronchus.

Future work includes (a) precise analysis about the stability of the proposed method, (b) test on real patients, and (c) development of a precise validation method.

Acknowledgments. The authors would like to thank Dr. Hiroshi Natori of Keiwakai Nishioka Hospital and Dr. Masaki Mori of Sapporo Kosei General Hospital for their advises. This work is supported by the program of the formation of innovation center for fusion of advanced technologies "Establishment of Early Preventing Medical Treatment Based on Medical Engineering for Analysis and Diagnosis" funded by the MEXT.

\section{References}

1. Mori, K., Deguchi, D., Sugiyama, J., et al.: Tracking of a bronchoscope using epipolar geometry analysis and intensity-based image registration of real and virtual endoscopic images. Medical Image Analysis 6, 321-336 (2002)

2. Schneider, A., Hautmann, H., Barfuss, H., et al.: Real-time image tracking of a flexible bronchoscope. In: Proc. of CARS 2004, vol. 1268, pp. 753-757 (2004)

3. Wegner, I., Vetter, M., Schoebinger, M., et al.: Development of a Navigation System for Endoluminal Brachytherapy in Human Lungs. In: Proc. of SPIE, vol. 6141, 614105-1 (2006)

4. Deligianni, F., Chung, A.J., Yang, G.Z.: Nonrigid 2-D/3-D Registration for Patient Specific Bronchoscopy Simulation With Statistical Shape Modeling: Phantom Validation. IEEE TMI 25(11), 1462-1471 (2006)

5. Kitasaka, T., Mori, K., Hasegawa, J., et al.: A Method for Extraction of Bronchus Regions from 3D Chest X-ray CT Images by Analyzing Structural Features of the Bronchus. FORMA 17(4), 321-338 (2002)

6. Nakada, K., Nakamoto, M., Sato, Y., et al.: A Rapid Method for Magnetic Tracker Calibration Using a Magneto-Optic Hybrid Tracker. In: Ellis, R.E., Peters, T.M. (eds.) MICCAI 2003. LNCS, vol. 2879, pp. 285-293. Springer, Heidelberg (2003) 